\title{
Damage localization in shear buildings by direct updating of physical properties
}

\author{
Alireza Entezami $\cdot$ Hashem Shariatmadar
}

Received: 6 August 2013/Accepted: 19 May 2014/Published online: 6 June 2014

(c) The Author(s) 2014. This article is published with open access at Springerlink.com

\begin{abstract}
The objective of this article is to present a new method for identifying the damage location in a multi-story shear building by direct model updating method. In this regard, structural perturbation matrices should be determined that are directly defined as the discrepancy between mass and stiffness matrices of undamaged and damaged structures. As a result of expanding the dynamic orthogonality conditions, mass and stiffness perturbation matrices are formulated by the initial information of undamaged structures as well as the structure's modal parameters before and after the occurrence of damages. These matrices cannot easily detect the damage site. Therefore, a more explicit determination of damage location is performed dividing the amount of change in these matrices' diagonals by the physical properties of undamaged structure. This modification facilitates the damage localization process and yields precise and preferable results in comparison with applying classical methods such as natural frequencies, mode shapes and structural properties changes. Subsequently, the applicability and effectiveness of the proposed damage detection method are verified numerically and experimentally. For numerical verification of the proposed methods, a six-story shear building is utilized as a discrete system. Then, the experimental verification of proposed methods is conducted detecting the location of damages in a simple laboratory frame. It can be deduced that the proposed damage localization method can reliably detect and also localize the structural damage.
\end{abstract}

\footnotetext{
A. Entezami $(\bowtie) \cdot H$. Shariatmadar

Department of Civil Engineering, Faculty of Engineering,

Ferdowsi University of Mashhad, Mashhad, Iran

e-mail: entezami@stu-mail.um.ac.ir

H. Shariatmadar

e-mail: shariatmadar@um.ac.ir
}

Keywords Damage localization - Shear building - Direct model updating method $\cdot$ Mass perturbation matrix . Stiffness perturbation matrix

\section{Introduction}

A great number of the structures, which were constructed several decades ago, are still in service and some of them have deteriorated. All over the world, many complex and large structures play important roles in transportation systems and social services. Hence, it is of great importance for researchers and civil engineers to monitor these structure's health and detect any symptoms of damages in them. Recently, a great deal of attention has been focused on structural health monitoring and damage identification based on vibration-based techniques. Vibration-based method has been developed and applied to detect structural damages based on modal analysis. According to this method, various damage identification algorithms have been developed for dealing with three key problems, i.e., detecting the presence of damages in the structure, detecting the locations of structural damages and estimating the damages' extent. Most of the existing methods can be thought of as two-stage algorithms in which damage locations are detected at first and then damage extents are estimated (Gomes and Silva 2008). The basic idea of vibration-based technique is that modal parameters are functions of the physical properties such as mass, stiffness and damping matrices of the structures. Therefore, the variations of these physical properties will cause changes in the modal parameters. Damage is considered to be as changes in structural parameters that adversely affect the structure's performance. Hence, damage may also be defined as any deviation in the original geometrical or 
material properties of the structures that may cause undesirable stresses, displacements or vibrations in it. These weaknesses and deviations may be due to cracks, loose bolts, broken welds, corrosion and fatigue (Khoshnoudian and Esfandiari 2011).

The finite element method (FEM) may also be used to detect and locate the damaged elements through model updating (An and Ou 2013; Jaishi and Ren 2006; Shiradhonkar and Shrikhande 2011; Wu and Li 2006). The basic concept of finite element model updating is that the measured and analytical modal data are unlikely to be equal due to the presence of noise in the measurements and model inadequacies (Lee and Eun 2009). Therefore, incompatibility between experimental and analytical models is solved by finite element model updating methods. An important section of model updating methods is to apply the structure's responses in frequency and time domains to update or correct the structural models. The main difficulties lie in uncertainties of FE modeling and errors related to modal testing (Lin 1990). Uncertainties in the FE model are caused by inaccurate physical parameters, non-ideal boundary conditions and structure's nonlinear properties. Nonetheless, application of this concept for damage detection is categorized based on the differences between undamaged and damaged structures. It should also be mentioned that the amounts of perturbation characterized in two states are considered as damage levels. Therefore, determination of error parameters between undamaged and damaged structures is a major aspect in damage detection method by finite element modal updating.

In recent years, a great deal of attention has been devoted by many researchers to detecting damage according to the data obtained from vibration measurement. A detailed and comprehensive overview of the vibration-based damage detection techniques has been presented in the literature (Doebling et al. 1998, 1996; Salawu 1997; Yan et al. 2007). Considering all of the levels of damage detection process, it can be stated that the damage localization is an important and basic step in this process. Therefore, many researchers have focused on identifying the damage location. Initially, a number of them attempted to localize the structural damage using measured natural frequencies. Cawley and Adams (Cawley and Adams 1979) introduced a simple method for identifying the damage location by structural natural frequencies. They indicated that the data obtained from vibrational measurement may make a single point for detecting the damage sites. Kim et al. (2003) presented a frequency-based damage detection (FBDD) method for locating the structural damage from changes of natural frequencies. The merit of applying the change of natural frequencies to detect damage site is its convenient measurement and high accuracy. However, the measurement of natural frequencies may not provide enough information for damage detection to relate the changes to a correct damage location. Therefore, other damage indicators such as mode shape alterations should also be considered in the damage localization process. In this regard, Shi et al. (2000) proposed a sensitivity- and statistical-based method to localize structural damage by directly using the incomplete mode shapes. They expanded multiple damage location assurance criterion (MDLAC) and used incomplete mode shape instead of modal frequency. Roy and Ray-Chaudhuri (2013) introduced a mathematical basis to show the correlation between a structural damage and a change in the fundamental mode shape and its derivatives. They achieved a perturbation approach which was able to locate the damage site by changes in the fundamental mode shape. Moreno-Garcia et al. (2014) utilized mode shape derivatives, such as rotations (first derivative), curvatures (second derivative) and, more recently, third and four derivatives to localize damage in composite plates. Moreover, Reddy and Swarnamani indicated the effectiveness of using wavelet transform for detection and localization of small damages. They used the rotational mode shapes of the damaged and undamaged plate-like structures as well as continuous wavelet transform to attain the spatially distributed wavelet coefficients that were able to identify the damage position on a square plate (Reddy and Swarnamani 2013). Furthermore, model updating method has been introduced as an applicable technique for damage detection in dynamic systems, particularly for damage localization. Accordingly, Yang and Chen (2009) proposed a new approach for estimating the mass and stiffness matrices. The main objective of their research was to determine the error in mass and stiffness matrices by structure's initial physical properties and modal data. Lee and Eun (2009; Lee et al. 2011) provided a new method for estimating the dynamic parameters by error matrix and model updating method. The finite element model updating technique presented in this study improves aforementioned approaches to detect the structural damages and develops classical damage detection methods including changes in natural frequencies, mode shapes and physical properties of structures. Indeed, applying fundamental concepts of model updating method may simply lead to a more precise identification of damage location.

The objective of this study is to locate damage in the shear buildings by direct model updating technique and vibrational measurement data. To attain this aim, real modal parameters such as natural frequencies and mode shapes are identified by two methods. For numerical models, the general eigenvalue problem is used to simulate 
modal data. In all of the simulation formulations, the damping matrix is considered to be proportional. Therefore, the results of simulation method are calculated as real data. On the other hand, measured modal data from experimental models are usually complex data. In general, these data are impractical for being used in vibration-based techniques and model updating method. Thus, real modal parameters are extracted from complex modes by some efficient mathematical equations. The proposed approach for damage localization is introduced based on perturbation matrices that are defined by the difference between mass and stiffness matrices of undamaged and damage structures. These perturbation matrices are determined by expanding dynamic orthogonality conditions. It can be seen that the original mass and stiffness perturbation matrices cannot exactly detect the damage locations. Therefore, stiffness damage indicator (DRS) and mass damage indicator (DRM) are defined based on ratio of diagonal changes of perturbation matrices to diagonal values in undamaged mass and stiffness matrices. This modification facilitates the damage localization process and provides more precise and preferable results in comparison with the classical methods such as using natural frequencies, mode shapes and structural properties changes. Consequently, a numerical model of a six-story shear building and an experimental model of a three-story laboratory frame are utilized to verify the proposed method. It can be deduced that the DRS and DRM yield simple, robust and reliable mathematical formulations that can be applied in the numerical and practical structure, particularly shear buildings.

\section{Structural modeling and identification of modal parameters}

Modal parameters are individual properties of a structure that are related to its structural and physical properties including mass, stiffness and damping. The measured modal parameters may be in the form of frequency response function (FRF) data or natural frequencies and mode shapes. In general, modal data are obtained through either experimental or operational modal testing (Ewins 2000; He and $\mathrm{Fu} 2001$ ). Although using modal analysis provides an insight in dynamics of structures, it has got some disadvantages such as uncertainties, contamination by noise, and the disability to measure the complete data. Therefore, the most appropriate approximation in many numerical vibration-based techniques is to employ the simulation approaches for identifying the modal parameters. The main method for this simulation is to solve the generalized eigenvalue problem (generalized eigenvalue problem) that is a combination of the physical properties and modal data.
To simulate the modal parameters, equation of motion for a linear, elastic and time-invariant structure that has $N$ degrees of freedoms (DOF) is expressed as

$M \ddot{x}(\mathrm{t})+C \dot{x}(\mathrm{t})+K x(\mathrm{t})=f(\mathrm{t})$

where $M, C$ and $K$ are the mass, damping and stiffness matrices of the structure, respectively. Furthermore, $f(\mathrm{t})$ is the vector of applied forces and $x(\mathrm{t})$ is the vector of structural responses. In numerical approaches, in which damping is assumed to be proportional, the modal characteristics are identified as follows:

$\left(K-\omega_{i}^{2} M\right) \varphi_{i}=0$

In this expression, $\varphi_{i}$ and $\lambda_{i}$ are mode shapes and eigenvalue $\left(\lambda=\omega^{2}\right)$ of the structure, respectively. Both of these two parameters are of the same order. In addition, $N$ represents the total number of structure's DOF. Based on complete modal parameters, Eq. (2) can be rewritten as:

$K \Phi=\Lambda M \Phi$

where $\Phi$ is the matrix of mode shapes $\varphi_{i}$ and $\Lambda$ is a diagonal matrix whose elements are $\omega_{i}^{2}$. In fact, expression (3) is the complete form of the simulation approach for modal identification. This expression is defined as the generalized eigenvalue problem. Based on the above equation, only with dynamic properties such as mass and stiffness matrices and also considering the proportional damping, the complete modal parameters can be achieved as real data. In the structural dynamics, the shear buildings are often categorized as discrete dynamic systems or lumped-mass structures. Thus, for a discrete system of $N$ DOF the mass and stiffness matrices can be determined as:

$M=\left[\begin{array}{cccc}M_{1} & & & 0 \\ & \ddots & & \\ & & M_{i-1} & \\ 0 & & & M_{i}\end{array}\right]$

$K=\left[\begin{array}{ccccc}\left(K_{1}+K_{2}\right) & -K_{2} & & & 0 \\ -K_{2} & & \ddots & & \\ & & & \left(K_{i-1}+K_{i}\right) & -K_{i} \\ 0 & & & -K_{i} & K_{i}\end{array}\right]$

As can be observed in Eq. (3), the modal parameters are obtained according to the physical properties of structure. In contrast, one of the most precious capabilities of the above equation is estimating the mass and stiffness matrices based on identified modal parameters. This is of great importance, particularly in the process of damage detection in experimental models. In fact, estimating the initial properties of structures is one of the first steps of damage detection process when using both vibration-based 
technique and model updating method. Hence, FEM provides numerous theoretical techniques for modeling discrete and continuous structural systems (Rao 2011). Moreover, these physical properties that are determined by FEM methods can be estimated by modal data obtained from experimental models.

Assuming that modal parameters may be real or complex data, there are various direct methods that can estimate initial physical properties of structures. Generally, it is recommended to use orthogonality conditions and state space approach for real and complex data, respectively (Luş et al. 2003). Taking into consideration that real mode shapes satisfy the mass normalization condition as $\varphi_{i}^{T} M \varphi_{i}=1$, the mass and stiffness matrices will be determined via measured modal data as follows:

$M_{x}=\sum_{i=1}^{N}\left(\left\{\varphi_{x}\right\}_{i}\left\{\varphi_{x}\right\}_{i}^{T}\right)^{-1}$

$K_{x}=M_{x}\left(\sum_{i=1}^{N}\left(\omega_{x}\right)_{i}^{2}\left\{\varphi_{x}\right\}_{i}\left\{\varphi_{x}\right\}_{i}^{T}\right) M_{x}$

Where $M_{x}$ and $K_{x}$ are the mass and stiffness matrices of experimental models, respectively. Moreover, $\varphi_{x}$ and $\omega_{x}$ denote the measured real mode shapes and natural frequencies, respectively. It should be noted here that the obtained mass and stiffness matrices are not always valid for every measured modal parameter. In other words, scaled and normalized modes are essential for utilizing these expressions even in the case of simple structures.

Furthermore, damping plays an important role in the simulation method while performing model analysis. If the damping matrix is either symmetric or proportional, modal parameters will be extracted as real data through solving the linear eigenvalue problem. Otherwise, modal data will be complex quantities. Complex modal parameters in either experimental or theoretical models indicate the presence of damping in the structures, which should be considered in the simulation method (Balmes 1997; Fuellekrug 2008; Neugebauer et al. 2010). Since complex modal data are impractical in the system identification and damage detection processes, real modal parameters should be extracted from complex modes. Hence, the complex eigenvalue can be formulated as follows:

$\lambda_{i}=-\xi_{i} \omega_{i} \mp j \omega_{i} \sqrt{1-\xi_{i}^{2}}$

where $\xi_{i}$ and $\omega_{i}$ are modal damping ratio and undamped natural frequency, respectively. Moreover, $j=\sqrt{ }-1$ is the complex element. For negligible imaginary values of complex modes, the undamped natural frequency and damping ratio are determined as:

$\omega_{i}=\sqrt{\alpha_{i}^{2}+\beta_{i}^{2}}$ $\zeta_{i}=\frac{-\alpha_{i}}{\sqrt{\alpha_{i}^{2}+\beta_{i}^{2}}}$

where $\alpha_{i}$ is the real part of complex eigenvalue quantity, and $\beta_{i}$ is the imaginary part of Eq. (8). As can be observed, Eq. (9) indicates that the natural frequencies are generally considered as the eigenvalue of undamped structures (structures of zero damping). As a result, dynamic responses of structures are determined as independent modes and therefore real mode shapes can be expressed as:

$\varphi_{i}=\psi_{i} \sqrt{2 j \omega_{i} \sqrt{1-\zeta_{i}^{2}}}$

\section{Damage localization}

Occurrence of damage in a structure alters some of its properties including mass, stiffness, damping and also the vibrational responses. Thus, it is viable to determine the existence and location of damages in a structure if its vibrational behavior is identified. In this study, damage localization process is carried out by direct model updating of the damaged physical properties. The direct model updating method is generally utilized to correct and update mass and stiffness matrices of theoretical models using measured modal parameters. Thus, the updating process leads to a similar dynamic behavior in theoretical and experimental models (Mottershead and Friswell 1993; Mottershead et al. 2011). In addition, the main merit of this method is that it makes use of simple mathematical equations and yields accurate results. Hence, a novel method of damage localization is presented to utilize the direct model updating technique and provide reliable and practical equations for this process. In this study, variances of mass and stiffness matrices are utilized to evaluate damage localization process. It is clear that these matrices play a significant role in analyzing the structure's dynamic behavior due to structural damages. Reduction of stiffness and increase in the mass matrix have got more effect in this process. In fact, these changes lead to adverse dynamic behavior and indicate that damage has occurred. According to fundamental concepts of finite element model updating technique, two perturbation matrices are generated that are related to the discrepancy between mass and stiffness matrices of undamaged and damaged structures. By using these perturbation matrices, structural damage is readily evaluated through some simple mathematical equations.

\section{Damage localization by stiffness changes}

In order to determine the stiffness reduction index, the stiffness perturbation matrix should be introduced as the 
difference between stiffness of undamaged and damaged structures as follows:

$\Delta K=K_{\mathrm{d}}-K_{\mathrm{u}}$

To attain this matrix, the mode shape orthogonality condition related to the damaged structure is expressed as:

$\varphi_{\mathrm{d}}^{\mathrm{T}} K_{\mathrm{d}} \varphi_{\mathrm{d}}=\Lambda_{\mathrm{d}}$

Where $\Lambda_{\mathrm{d}}$ and $\varphi_{\mathrm{d}}$ are eigenvalue diagonal matrix and eigenvector (mode shape) of damaged structure, respectively. Damage in a structure leads to changes in mode shapes and natural frequencies, which can be expressed as follows:

$\Delta \Phi=\sum_{i=1}^{N}\left(\varphi_{\mathrm{d}}\right)_{i}-\left(\varphi_{\mathrm{u}}\right)_{i}$

$\Delta \Lambda=\sum_{i=1}^{N}\left(\lambda_{\mathrm{d}}\right)_{i}-\left(\lambda_{\mathrm{u}}\right)_{i}=\sum_{i=1}^{N}\left(\omega_{\mathrm{d}}^{2}\right)-\left(\omega_{\mathrm{u}}^{2}\right)_{i}$

In these expressions, the subscripts $\mathrm{u}$ and $\mathrm{d}$ indicate the undamaged and damaged structures, respectively. Furthermore, $N$ is the total number of DOF. After substituting these linear objective functions as well as Eq. (12) into Eq. (13) it can be rewritten as:

$\left(\varphi_{\mathrm{u}}+\Delta \varphi\right)^{\mathrm{T}}\left(K_{\mathrm{u}}+\Delta K\right)\left(\varphi_{\mathrm{u}}+\Delta \varphi\right)=\Lambda_{\mathrm{d}}$

which can be expanded to yield:

$$
\begin{aligned}
& \left(\varphi_{\mathrm{u}}^{\mathrm{T}} K_{\mathrm{u}} \varphi_{\mathrm{u}}\right)+\left(\varphi_{\mathrm{u}}^{\mathrm{T}} K_{\mathrm{u}} \Delta \varphi\right)+\left(\varphi_{\mathrm{u}}^{\mathrm{T}} \Delta K \varphi_{\mathrm{u}}\right)+\left(\varphi_{\mathrm{u}}^{\mathrm{T}} \Delta K \Delta \varphi\right) \\
& \quad+\left(\Delta \varphi^{\mathrm{T}} K_{\mathrm{u}} \varphi_{\mathrm{u}}\right)+\left(\Delta \varphi^{\mathrm{T}} K_{\mathrm{u}} \Delta \varphi\right)+\left(\Delta \varphi^{\mathrm{T}} \Delta K \varphi_{\mathrm{u}}\right)+\left(\Delta \varphi^{\mathrm{T}} \Delta K \Delta \varphi\right)=\Lambda_{\mathrm{d}}
\end{aligned}
$$

Neglecting the higher order terms, the preceding equation reduces to:

$\varphi_{\mathrm{u}}^{\mathrm{T}} \Delta K \varphi_{\mathrm{u}}=\Lambda_{\mathrm{d}}-\Lambda_{\mathrm{u}}-\left(\varphi_{\mathrm{u}}^{\mathrm{T}} K_{\mathrm{u}} \Delta \varphi\right)-\left(\Delta \varphi^{\mathrm{T}} K_{\mathrm{u}} \varphi_{\mathrm{u}}\right)$

By pre- and post-multiplying the Eq. (18) by $\varphi_{\mathrm{u}}^{\mathrm{T}}$ and $\varphi_{\mathrm{u}}$, respectively, we will have:

$\varphi_{\mathrm{u}} \varphi_{\mathrm{u}}^{\mathrm{T}} \Delta K \varphi_{\mathrm{u}} \varphi_{\mathrm{u}}^{\mathrm{T}}=\varphi_{\mathrm{u}}\left(\Lambda_{\mathrm{d}}-\Lambda_{\mathrm{u}}-\left(\varphi_{\mathrm{u}}^{\mathrm{T}} K_{\mathrm{u}} \Delta \varphi\right)-\left(\Delta \varphi^{\mathrm{T}} K_{\mathrm{u}} \varphi_{\mathrm{u}}\right)\right) \varphi_{\mathrm{u}}^{\mathrm{T}}$

It is clear that the right-hand side of Eq. (19) is an $(N \times N)$ matrix that pertains to the intact stiffness matrix and modal parameters of both undamaged and damaged structures. Therefore, a matrix called stiffness error coefficient is defined for compressing the above equation as follows:

$C_{\mathrm{k}}=\Lambda_{\mathrm{d}}-\Lambda_{\mathrm{u}}-\left(\varphi_{\mathrm{u}}^{\mathrm{T}} K_{\mathrm{u}} \Delta \varphi\right)-\left(\Delta \varphi^{\mathrm{T}} K_{\mathrm{u}} \varphi_{\mathrm{u}}\right)$

Since mode shape vectors have already been normalized by the mass matrix as $\varphi_{\mathrm{u}}^{\mathrm{T}} M_{\mathrm{u}} \varphi_{\mathrm{u}}=I$, multiplication of undamaged mode shape matrices is equivalent to the inverse intact mass matrix. Therefore, the expression $\varphi_{\mathrm{u}} \varphi_{\mathrm{u}}^{\mathrm{T}}=M_{\mathrm{u}}^{-1}$ is substituted into Eq. (18) and the stiffness perturbation matrix is described as follows:

$\Delta K=M_{\mathrm{u}} \varphi_{\mathrm{u}} C_{\mathrm{k}} \varphi_{\mathrm{u}}^{\mathrm{T}} M_{\mathrm{u}}$

As can be seen, direct model updating of physical properties leads to the determination of damage index according to stiffness reduction. It is apparent that the proposed equation is formulated only by small amount of available structural information such as the undamaged mass and stiffness matrices and also modal parameters before and after the damage. Although, Eq. (21) provides some general information regarding changes of the stiffness matrices in undamaged and damaged structures, initial values of stiffness perturbation matrix cannot exactly detect the damage locations. Therefore, diagonal changes of the stiffness discrepancy matrix $(\Delta K)$ are utilized to find its maximum values. This modification leads to a simpler damage localization process in comparison with Eq. (21). Hence, the stiffness damage localization indicator (DRS), which is defined as diagonal values of the stiffness perturbation matrix, is presented as follows:

$\mathrm{DRS}=\sum_{i=1}^{N}\left(\frac{\Delta k}{k_{\mathrm{u}}}\right)_{i}$

Where $\Delta k_{i}$ and $k_{\mathrm{u} i}$ are the diagonal values of discrepancy stiffness matrix $\Delta K$ and stiffness of undamaged structure $K_{\mathrm{u}}$, respectively. It is clear that the proposed method detects damage location simply and more precisely than Eq. (21). Indeed, this method can remove the complications of stiffness perturbation matrix, particularly the confusion present in its data. Therefore, dividing the changes in diagonal values of $\Delta K$ by the corresponding quantities in undamaged stiffness matrix will lead to determination of maximum values of DRS.

\section{Damage localization by mass changes}

On the whole, the increase of mass in the structures cannot be directly considered as structural damage. However this change sometimes leads to alterations in the structure's dynamic performance. For instance, presence of excessive masses or adding heavy masses to the structure may cause considerable structural damages when earthquake or huge vibrational loadings are applied to the structure. Indeed, the seismic forces are induced in the heavy masses present at various stories. Therefore, higher amounts of mass present in the structure lead to higher seismic forces and it can be noticed that heavier buildings attract larger seismic forces and so are prone to damage. Thus, this study attempts to probe the dynamic behavior of shear building when masses are added to floor levels. 
As indicated in the prior section, the changes of mass were neglected due to their low influence in comparison with stiffness alterations. Here, the effect of mass matrix is considered for identifying the damage locations. Similar to the previous section, the mass perturbation matrix should be determined. This matrix can be determined via expanding the mass orthogonal condition for damaged structure as follows:

$\varphi_{\mathrm{d}}^{\mathrm{T}} M_{\mathrm{d}} \varphi_{\mathrm{d}}=I$

To achieve the mass perturbation matrix, changes of mode shapes and natural frequencies (Eqs. 14 and 15) as well as the mass alteration $\left(\Delta M=M_{\mathrm{d}}-M_{\mathrm{u}}\right)$ are substituted into Eq. (23) as:

$\left(\varphi_{\mathrm{u}}+\Delta \varphi\right)^{\mathrm{T}}\left(M_{\mathrm{u}}+\Delta M\right)\left(\varphi_{\mathrm{u}}+\Delta \varphi\right)=I$

By expanding the above equation and neglecting its higher order terms, the final equation for mass perturbation matrix can be formulated as follows:

$\varphi_{\mathrm{u}}^{\mathrm{T}} \Delta M \varphi_{\mathrm{u}}=-\Delta \varphi^{\mathrm{T}} M_{\mathrm{u}} \varphi_{\mathrm{u}}-\varphi_{\mathrm{u}}^{\mathrm{T}} M_{\mathrm{u}} \Delta \varphi$

Then, the mass perturbation matrix can be multiplied by $\varphi_{\mathrm{u}}^{\mathrm{T}}$ and $\varphi_{\mathrm{u}}$ and rewritten as:

$\varphi_{\mathrm{u}} \varphi_{\mathrm{u}}^{\mathrm{T}} \Delta M \varphi_{\mathrm{u}} \varphi_{\mathrm{u}}^{\mathrm{T}}=\varphi_{\mathrm{u}}\left(-\Delta \varphi^{\mathrm{T}} M_{\mathrm{u}} \varphi_{\mathrm{u}}-\varphi_{\mathrm{u}}^{\mathrm{T}} M_{\mathrm{u}} \Delta \varphi\right) \varphi_{\mathrm{u}}^{\mathrm{T}}$

The right-hand side of Eq. (26) is an $N \times N$ matrix that consists of the undamaged mass matrix and corresponding modal parameters before and after occurrence of structural damages. This equation can be described as the mass error coefficient matrix:

$C_{\mathrm{m}}=-\Delta \varphi^{\mathrm{T}} M_{\mathrm{u}} \varphi_{\mathrm{u}}-\varphi_{\mathrm{u}}^{\mathrm{T}} M_{\mathrm{u}} \Delta \varphi$

As discussed before, if mode shapes satisfy the mass normalization condition, expression $\varphi_{\mathrm{u}} \varphi_{\mathrm{u}}^{\mathrm{T}}=M_{\mathrm{u}}^{-1}$ is inserted into Eq. (26) and the final equation for attaining the mass perturbation matrix can be expressed as:

$\Delta M=M_{\mathrm{u}} \varphi_{\mathrm{u}} C_{\mathrm{m}} \varphi_{\mathrm{u}}^{\mathrm{T}} M_{\mathrm{u}}$

It is clear that the formulation of Eq. (28) is similar to the stiffness perturbation matrix. This equation entirely explains the adverse effect of mass alterations in the buildings. To determine the damage locations more precisely, diagonal changes of the mass discrepancy matrix $(\Delta M)$ are utilized to find its maximum values, exactly similar to pervious section. Therefore, the ratio of diagonal values of $\Delta M$ to the corresponding values in the undamaged mass matrix can be defined as a new damage localization index as follow:

$\mathrm{DRM}=\sum_{i=1}^{N}\left(\frac{\Delta m}{m_{\mathrm{u}}}\right)_{i}$

In this equation, DRM is a relative indicator of damage index based on mass changes. This indicator is a vector that its maximum value indicates the damage locations. $\Delta m_{i}$ and $m_{\mathrm{u} i}$ are diagonal values of mass perturbation and intact mass matrix, respectively.

\section{Numerical investigation}

To investigate the effectiveness of proposed damage detection algorithm, a six-story shear building was considered as shown in Fig. 1. Formulation of discrete systems was carried out to generate mass and stiffness matrices of this shear building (Rao 2011). It was assumed that the slabs are confined amongst beams and behave as rigid body; hence, the stiffness of each story is computed summing the stiffness of columns. Furthermore, mass of each story was calculated summing half weight of the top and bottom walls as well as slab's weight. After determining structural properties, natural frequencies and mode shapes of the shear building were computed using Eq. (3). Due to considering the proportional damping, modal parameters were obtained as real values which do not need any transfer functions for being extracted from complex modes. The initial physical properties of the shear building are presented in Table 1.

Several damage cases are considered to investigate the effectiveness of proposed damage localization methods. Specifications of these cases are summarized in Table 2.

Induced damage cases change the properties of the shear building. In practice, the identified modal frequencies are more accurate than mode shapes; hence, it is preferred to use natural frequency alterations for investigating the structure's dynamic behavior. Natural frequencies of the damaged structure are presented in Table 3.

As can be noticed, damages cause decrease in natural frequencies and also adverse vibrational performances. As a result of these induced damages adverse dynamic behavior occurs in the structure. Locations of induced damages are initially determined using the proposed DRS and DRM methods and obtained data such as initial physical properties and modal parameters of the structure before and after the occurrence of damage. Figures 2, 3, and 4 show the damage locations detected in the shear building.

As shown in these figures, location of damaged stories can be detected using direct model updating of physical properties as the stiffness reduction and the mass increase. Figures $2 \mathrm{a}, \mathrm{b}$ and $3 \mathrm{a}, \mathrm{b}$ illustrate the damage locations detected by DRS. As can be observed, damage sites are accurately identified when single or multiple damage cases are present. In these figures, the highest peaks of DRS diagram indicate the locations of damage. As a 
Fig. 1 a Six-story shear building; b the six-story shear building simulated as a discrete system

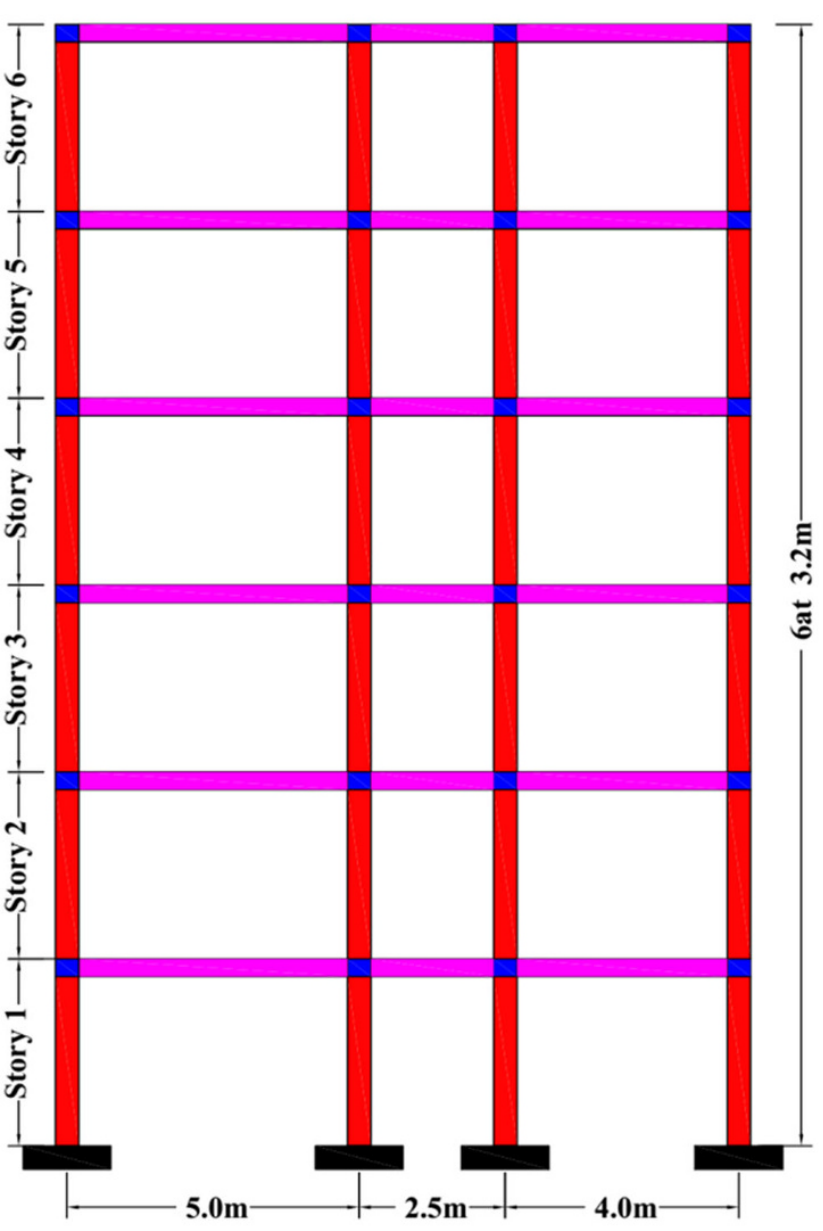

(a)

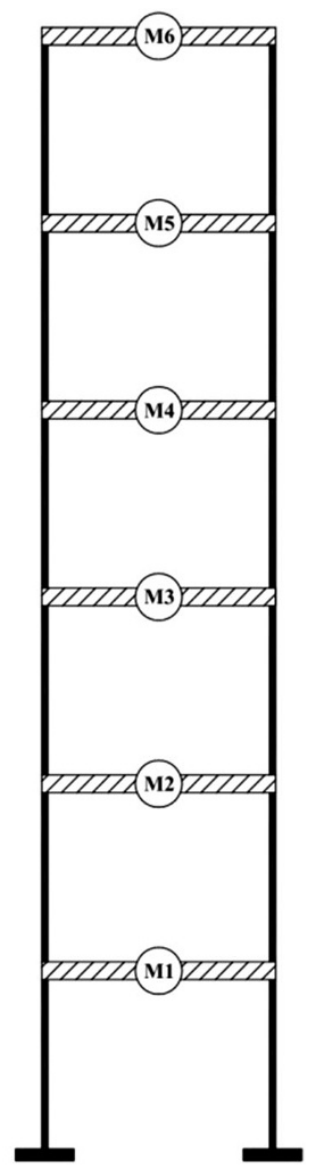

(b)
Table 1 Physical properties of six-story shear building

\begin{tabular}{lrrrrrr}
\hline $\begin{array}{l}\text { Story } \\
\text { number }\end{array}$ & $\begin{array}{l}\text { Story } \\
1\end{array}$ & $\begin{array}{l}\text { Story } \\
2\end{array}$ & $\begin{array}{l}\text { Story } \\
3\end{array}$ & $\begin{array}{l}\text { Story } \\
4\end{array}$ & $\begin{array}{l}\text { Story } \\
5\end{array}$ & $\begin{array}{l}\text { Story } \\
6\end{array}$ \\
\hline $\begin{array}{l}\text { Mass (ton) } \\
\text { Stiffness } \\
(\text { ton/m) }\end{array}$ & 10 & 10 & 10 & 8 & 8 & 6 \\
\hline
\end{tabular}

matter of fact, in the case of single damage, the tallest column in DRS diagram points out the damaged story. Figure $4 \mathrm{a}, \mathrm{b}$ shows the mass increase in the shear building that causes inappropriate dynamic behavior. According to these figures, the DRM is more simply determined in comparison with DRS due to simplicity of mass matrix and related formulations of damage index by mass increase. Provided that the initial data of structural modeling is more precise, the model updating process will yield preferable and more precise results in damage localization. In other words, accurate and reliable data for modeling the structure leads to more appropriate results in damage detection.
Table 2 Damage induced in the six-story shear building

\begin{tabular}{llll}
\hline Case number & Story no. & \multicolumn{2}{l}{ Damage index } \\
\cline { 3 - 4 } & & Mass (\%) & Stiffness (\%) \\
\hline 1 & 1 & - & -40 \\
2 & 1 & - & -30 \\
& 6 & - & -20 \\
3 & 3 & - & -30 \\
4 & 3 & - & -10 \\
& 4 & - & -20 \\
5 & 1 & 50 & - \\
6 & 3 & 25 & - \\
& 5 & 30 & - \\
\hline
\end{tabular}

\section{Experimental evaluation}

Damage in a three-story laboratory frame was investigated in this part of study. This frame, which is $2.1-\mathrm{m}$ high, was constructed using three steel plates of $650 \times 650 \times 5 \mathrm{~mm}^{3}$ and four equal L-shaped aluminum 
columns with a $30 \times 30 \mathrm{~mm}$ cross-section. Steel plates and columns at each story were bolted with aluminum brackets as shown in Fig. 5. Columns' thickness was

Table 3 Natural frequencies of the six-story shear building in the undamaged and damaged cases

\begin{tabular}{lcccccc}
\hline $\begin{array}{l}\text { Damage } \\
\text { case }\end{array}$ & $\begin{array}{c}\text { Mode } \\
1\end{array}$ & $\begin{array}{c}\text { Mode } \\
2\end{array}$ & $\begin{array}{c}\text { Mode } \\
3\end{array}$ & $\begin{array}{c}\text { Mode } \\
4\end{array}$ & $\begin{array}{c}\text { Mode } \\
5\end{array}$ & $\begin{array}{c}\text { Mode } \\
6\end{array}$ \\
\hline Undamaged & 0.9230 & 2.4558 & 3.9743 & 5.0845 & 6.1236 & 6.5575 \\
Case 1 & 0.8404 & 2.2819 & 3.7944 & 4.9722 & 6.0360 & 6.5243 \\
Case 2 & 0.8665 & 2.3047 & 3.7181 & 4.8031 & 5.9478 & 6.4891 \\
Case 3 & 0.8826 & 2.4483 & 3.7205 & 4.9907 & 6.0065 & 6.3854 \\
Case 4 & 0.8961 & 2.3830 & 3.8889 & 4.8763 & 6.0742 & 6.3450 \\
Case 5 & 0.9182 & 2.3693 & 3.7182 & 4.8515 & 5.9002 & 6.4861 \\
Case 6 & 0.8697 & 2.3534 & 3.9258 & 4.7985 & 5.8989 & 6.3442 \\
\hline
\end{tabular}

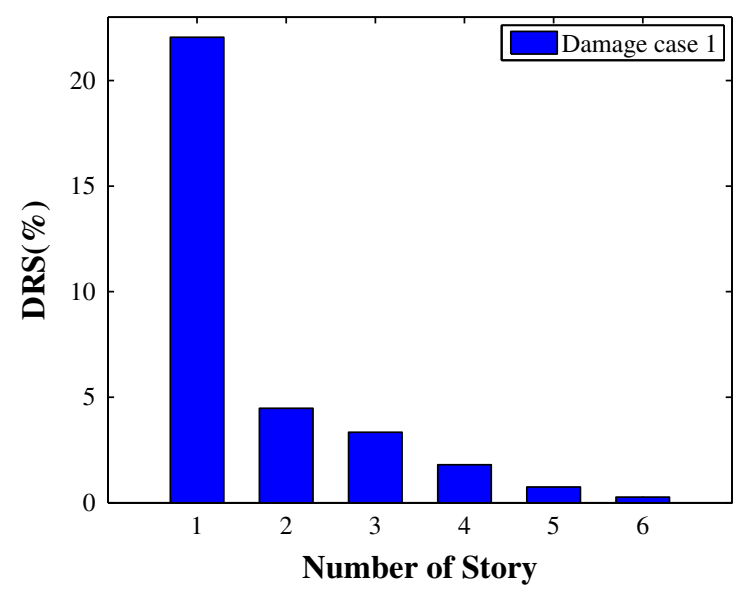

(a) considered to be equal to 5 and $3 \mathrm{~mm}$ in undamaged and damaged states, respectively. In order to simulate the localized damage at different stories, each column was made of three separate $0.7-\mathrm{m}$-long segments instead of one long L-shaped part. These separate segments can be easily replaced by new ones. For damage detection, the laboratory frame was subjected to experimental modal test in the structural dynamics laboratory. The excitation force was applied by a force impact hammer of Brüel \& Kjær A8202 type. Each floor was equipped with a Brüel \& Kjær 4370 type accelerometer in the $x$-direction. Signals from the accelerometers were analyzed to identify natural frequencies and mode shapes. Tables 4 and 5 indicate the initial modal data of undamaged frame.

Existence of damping in the structure causes the modal parameters to be identified as complex data. As

Fig. 2 Damage localization in the six-story shear building by DRS: a damage scenario 1, b damage scenario 2

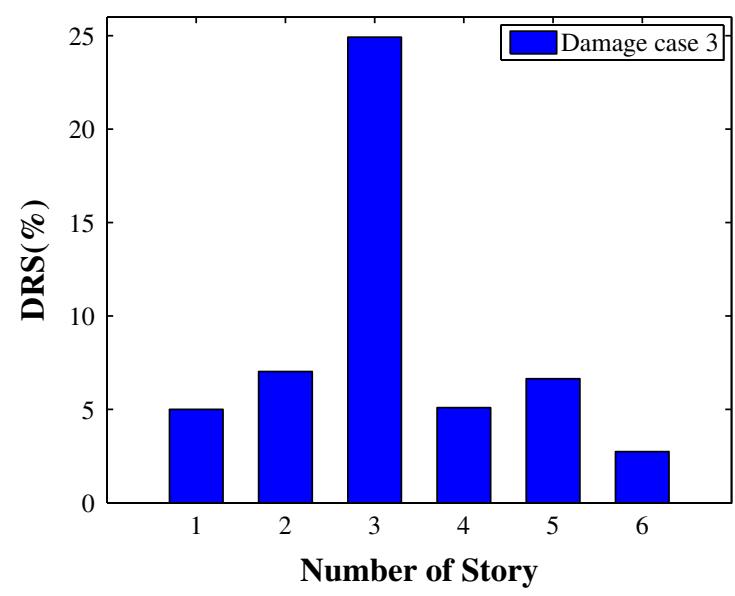

(a)

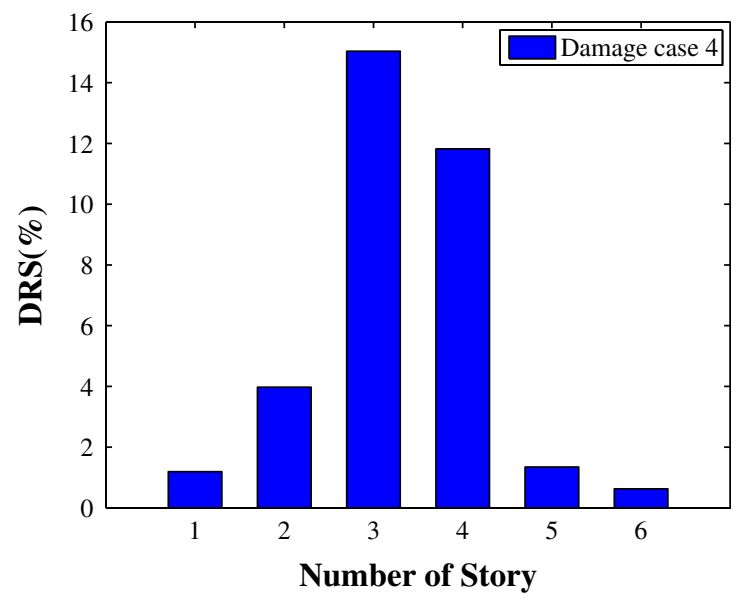

(b)

Fig. 3 Damage localization in the six-story shear building by DRS: a damage scenario 3, b damage scenario 4 


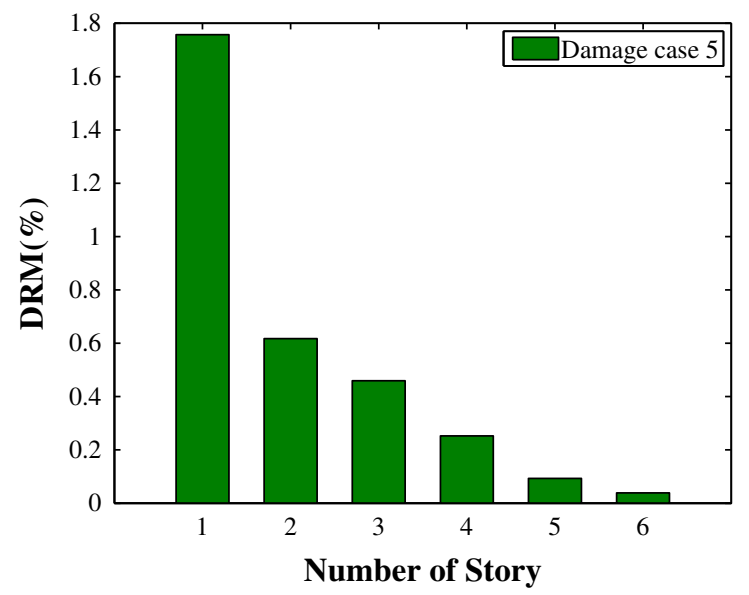

(a)

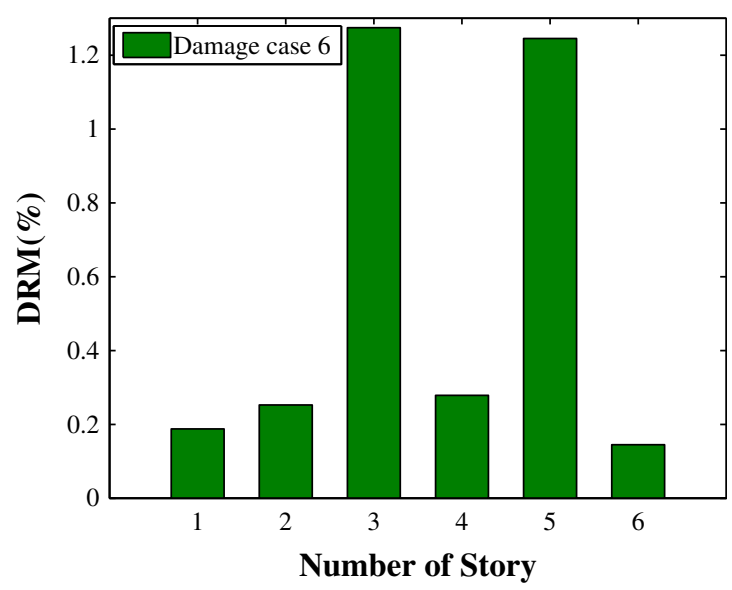

(b)

Fig. 4 Damage localization in the six-story shear building by DRM: a damage scenario 5, b damage scenario 6

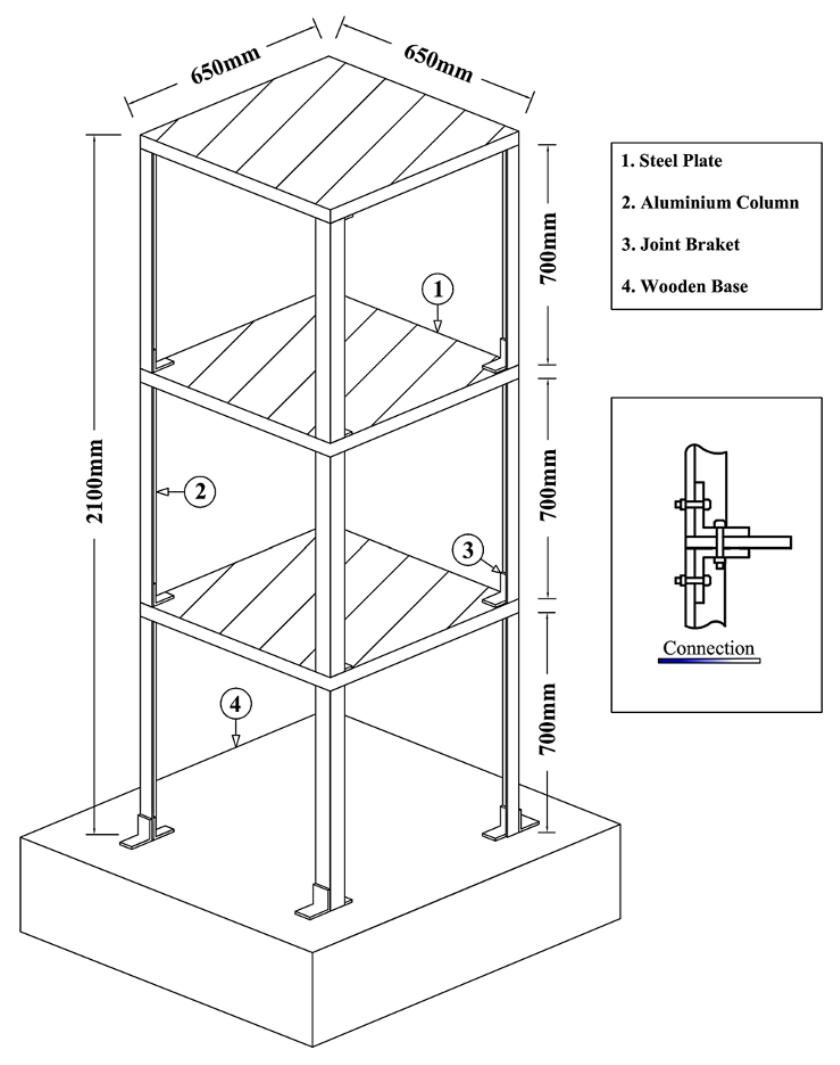

Fig. 5 The three-story laboratory frame used for the study

can be seen in Tables 4 and 5, the imaginary parts of these modal data are negligible. In the proposed damage detection method, real modal data are required for formulations. Therefore, Eqs. (8)-(10) are used to extract the real parts from complex modal
Table 4 Identified complex eigenvalues and damping ratios of the three-story laboratory frame

\begin{tabular}{lccl}
\hline Stories & $\begin{array}{l}\text { Complex } \\
\text { eigenvalues }(\mathrm{Hz})\end{array}$ & $\begin{array}{l}\text { Complex eigenvalues } \\
(\mathrm{rad} / \mathrm{s})\end{array}$ & $\begin{array}{l}\text { Damping } \\
\text { ratios }(\%)\end{array}$ \\
\hline First & $6.76 \pm 0.2 j$ & $42.5077 \pm 1.4502 j$ & $0.58 \pm 0.25 j$ \\
Second & $17.55 \pm 2.4 j$ & $110.1869 \pm 14.9537 j$ & $0.66 \pm 0.22 j$ \\
Third & $26.15 \pm 4.5 j$ & $164.2910 \pm 28.5367 j$ & $0.82 \pm 0.41 j$ \\
\hline
\end{tabular}

parameters. Obtained results are summarized in Tables 6 and 7.

In the proposed damage detection method, mass and stiffness matrices of undamaged structure must be determined. Thus, initial properties of the laboratory frame are calculated using the real measured modal data. Amounts of mass and stiffness evaluated for different stories of the laboratory frame are presented in Tables 8 and 9, respectively.

It should be noted that determining the mass and stiffness matrices is an inevitable and essential part in the proposed damage detection approach. It is of great significance that incorrect estimation of the structure's initial physical properties leads to improper damage detection. Thus, dynamic responses of experimental and numerical models are compared to ensure the accuracy in estimation of mass and stiffness matrices. This process is carried out by comparing the amounts of experimental natural frequencies with estimated ones.

Table 10 indicates that the initial physical properties are accurately estimated since the discrepancy between experimental and estimated natural frequencies is $<3 \%$. Based on the identified mass and stiffness matrices for 
Table 5 Complex eigenvectors of the three-story laboratory frame

\begin{tabular}{llrr}
\hline Stories & \multicolumn{1}{l}{ Mode 1 } & \multicolumn{1}{c}{ Mode 2 } & \multicolumn{1}{c}{ Mode 3 } \\
\hline First & $0.0894 \pm 0.0071 j$ & $0.2129 \pm 0.0151 j$ & $0.6835 \pm 0.0222 j$ \\
Second & $0.4204 \pm 0.0312 j$ & $0.5613 \pm 0.0761 j$ & $-0.2317 \pm 0.0105 j$ \\
Third & $0.6281 \pm 0.0597 j$ & $-0.4460 \pm 0.0165 j$ & $0.0561 \pm 0.0077 j$ \\
\hline
\end{tabular}

Table 6 Real mode shapes of the three-story laboratory frame

\begin{tabular}{llrr}
\hline Stories & Mode 1 & Mode 2 & \multicolumn{1}{c}{ Mode 3 } \\
\hline First & 0.0896 & 0.2131 & 0.6338 \\
Second & 0.4215 & 0.5664 & -0.2320 \\
Third & 0.6309 & -0.4463 & 0.0566 \\
\hline
\end{tabular}

Table 7 Real natural frequencies of the three-story laboratory frame

\begin{tabular}{llll}
\hline Mode number & Mode 1 & Mode 2 & Mode 3 \\
\hline Natural frequencies & 42.5387 & 111.2131 & 166.7371
\end{tabular}

Table 8 Mass values of the three-story laboratory frame

\begin{tabular}{llll}
\hline Stories & Story 1 & Story 2 & Story 3 \\
\hline Mass $(\mathrm{Kg})$ & 1.9188 & 1.8108 & 1.6654 \\
\hline
\end{tabular}

Table 9 Stiffness values of the three-story laboratory frame

\begin{tabular}{llll}
\hline Stories & Story 1 & Story 2 & Story 3 \\
\hline Stiffness $(\mathrm{N} / \mathrm{m})$ & 40,099 & 9,891 & 9,078 \\
\hline
\end{tabular}

Table 10 Amounts of experimental and estimated natural frequencies

\begin{tabular}{llll}
\hline Mode number & Mode 1 & Mode 2 & Mode 3 \\
\hline Experimental frequencies & 42.5387 & 111.2131 & 166.7371 \\
Estimated frequencies & 41.8601 & 110.7166 & 163.3627 \\
\hline
\end{tabular}

undamaged laboratory frame, three damage cases were considered for the modal test. It should also be mentioned that separate modal tests were carried out via impact hammer on the frame for each damage case. In the first case, a $0.5-\mathrm{kg}$ steel plate was stuck to second story. In the second damage case, columns of the first story were replaced by $30 \times 30 \mathrm{~mm}$ L-shaped aluminum columns of 3 -mm thickness. In this case, damage is introduced as a reduction in column's thickness and so the frame's stiffness is reduced. In damage case 2 , in addition to the first story,
Table 11 Natural frequencies evaluated in the three-story laboratory frame in different damage cases

\begin{tabular}{llrr}
\hline Damage case & Mode 1 & Mode 2 & Mode 3 \\
\hline Case 1 & 40.7215 & 103.8334 & 165.1451 \\
Case 2 & 41.3037 & 108.3116 & 152.7000 \\
Case 3 & 39.9300 & 97.6946 & 151.6575 \\
\hline
\end{tabular}

columns of the third story were also replaced by L-shaped columns as described in the previous case. The identified natural frequencies for all damage cases are listed in Table 11.

In the present investigation, the values of both DRM and DRS are experimentally determined to detect the damage sites using the proposed direct model updating method. Figure 6 demonstrates the location of damage in the first damage case by DRM. In other words, the tallest column of DRM diagram indicates that damage is occurred in the second story due to attaching the steel plate to this story. Moreover, as depicted in Figs. 7 and 8, damage sites are identified in the first and third stories' columns, since they were selected to detect locations of induced damage. As can be seen in the first damage scenario, the tallest column of DRS diagram shows the identified damage site. It is clear that the first and third stories have got higher peaks in comparison with the undamaged story. Consequently, it can be deduced that the proposed damage localization method is able to detect damage in multiple damage cases without making use of sensitivity analysis. Although, utilizing the DRM is simpler than DRS, the final results of stiffness damage index have more influence on the dynamic performance of shear buildings. For this reason, the stiffness changes in the third damage scenario are more remarkable than those of the first and second damage scenario; i.e., in the third damage case, a considerable alteration in the stiffness components of the second story is observed in addition to that of the first and third stories. It can be deduced that stiffness changes have taken place in the second story due to the presence of connections between members of the second story and damaged stories. As a result, surveying the structural connections of each story such as beamcolumn joints in the shear buildings has a significant influence on transmission of damage to other parts of structures. 


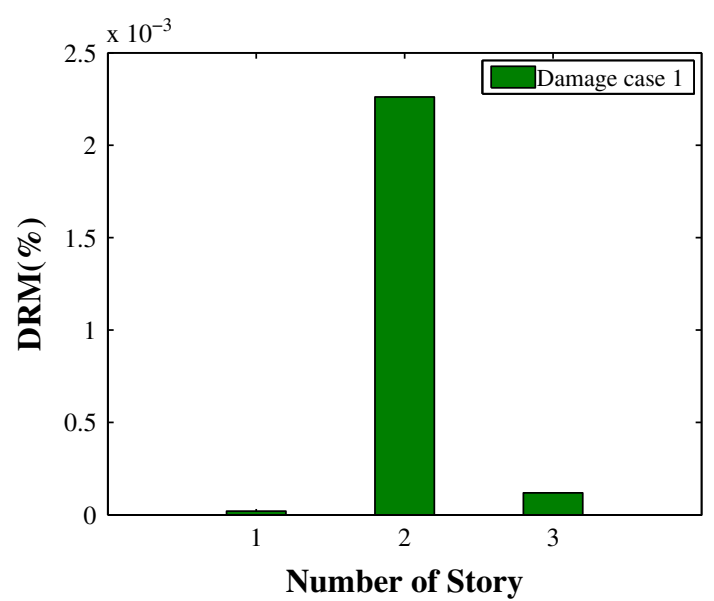

Fig. 6 Damage localization in the laboratory frame by DRM: damage case 1

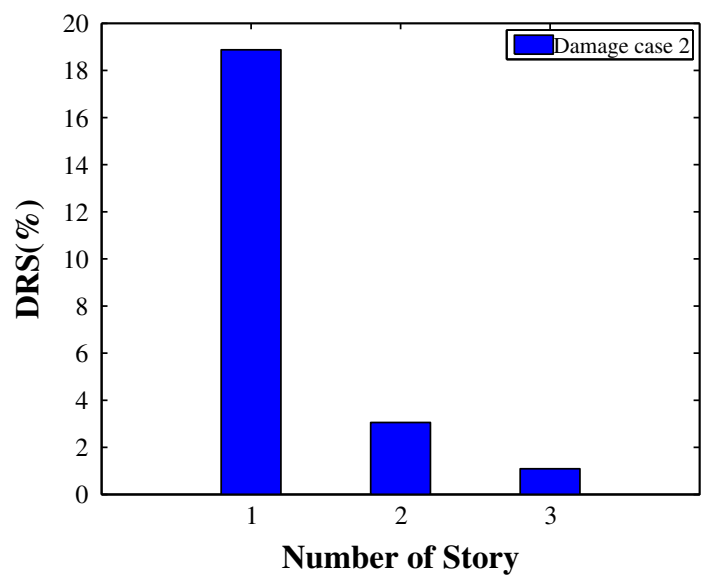

Fig. 7 Damage localization in the laboratory frame by DRS: damage case 2

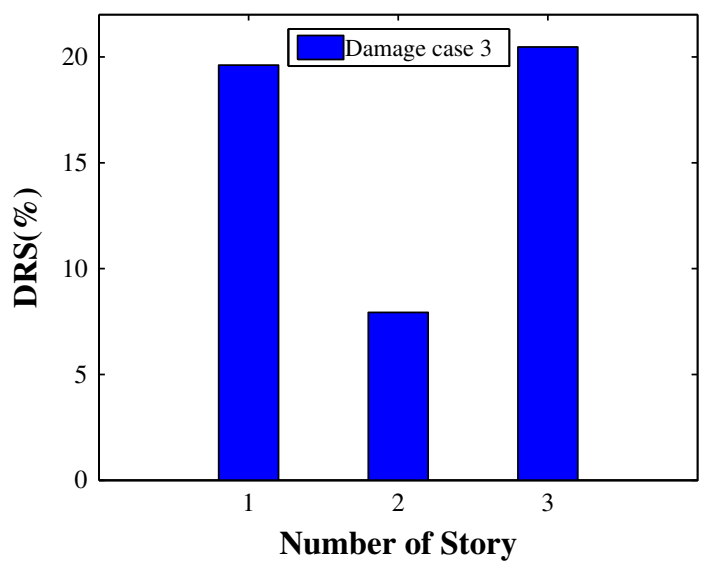

Fig. 8 Damage localization in the laboratory frame by DRS: damage case 3

\section{Conclusion}

In this study, a new proposed damage detection method is introduced for assessing the structural damage in the shear buildings. At the first stage of this method, location of damaged elements is identified by a direct model updating of physical properties which is defined as the stiffness DRS. Subsequently, the effect of mass increase is evaluated as a damage index. The proposed damage localization method requires using mode shapes and natural frequencies. Therefore, generalized eigenvalue problem is numerically used assuming that the damping is proportional. In order to assess the proposed method, a three-story laboratory frame is tested by impact hammer modal testing. The extracted modal data from experimental modal testing are usually complex values. Since real modal parameters play an important role in model updating method, real modes are extracted from complex data. For identifying the damage locations, mass and stiffness perturbation matrices should be determined. These matrices are related to physical properties of structure and are ordinarily unknown. Therefore, the generalized and mass normalization condition for damaged structures is expanded. As a result of expanding these mentioned equations, the perturbation matrices are determined only by undamaged mass and stiffness matrices as well as modal parameters before and after of damages. Finally, the applicability and effectiveness of the proposed damage detection method is verified both numerically and experimentally. For numerically verifying the proposed method, a six-story shear building is modeled as a discrete system. Then, the experimental verification of proposed method is conducted detecting the location of damages in a simple laboratory frame. The results obtained from these two verification studies indicate that the damage localization approach can exactly identify the damage sites. It should be mentioned that results of DRM and DRS depend on correct determination of the structural model's initial information.

Open Access This article is distributed under the terms of the Creative Commons Attribution License which permits any use, distribution, and reproduction in any medium, provided the original author(s) and the source are credited.

\section{References}

An Y, Ou J (2013) Experimental and numerical studies on model updating method of damage severity identification utilizing four cost functions. Struct Control Health Monit 20:107-120

Balmes E (1997) New results identification normal modes from experimental complex modes. Mech Syst Signal Process 11:229-243

Cawley P, Adams R (1979) The location of defects in structures from measurements of natural frequencies. J Strain Anal Eng Des $14: 49-57$ 
Doebling SW, Farrar CR, Prime MB, Shevitz DW (1996) Damage identification and health monitoring of structural and mechanical systems from changes in their vibration characteristics: a literature review. In: Other information: PBD: May 1996

Doebling SW, Farrar CR, Prime MB (1998) A summary review of vibration-based damage identification methods. Shock Vib Dig 30:91-105

Ewins DJ (2000) Modal testing: theory and practice and application, 2nd edn. Wiley, USA

Fuellekrug U (2008) Computation of real normal modes from complex eigenvectors. Mech Syst Signal Process 22:57-65

Gomes HM, Silva NRS (2008) Some comparisons for damage detection on structures using genetic algorithms and modal sensitivity method. Appl Math Model 32:2216-2232

He J, Fu ZF (2001) Modal analysis, 1st edn. Butterworth-Heinemann, UK

Jaishi B, Ren W-X (2006) Damage detection by finite element model updating using modal flexibility residual. $\mathrm{J}$ Sound Vib 290:369-387

Khoshnoudian F, Esfandiari A (2011) Structural damage diagnosis using modal data. Sharif Univ Technol 18:853-860

Kim J-T, Ryu Y-S, Cho H-M, Stubbs N (2003) Damage identification in beam-type structures: frequency-based method vs modeshape-based method. Eng Struct 25:57-67

Lee E-T, Eun H-C (2009) Update of corrected stiffness and mass matrices based on measured dynamic modal data. Appl Math Model 33:2274-2281

Lee E-T, Rahmatalla S, Eun H-C (2011) Estimation of parameter matrices based on measured data. Appl Math Model $35: 4816-4823$

Lin CS (1990) Location of modeling error using modal test data. AIAA J 28:1650-1654

Luş H, De Angelis M, Betti R, Longman R (2003) Constructing secondorder models of mechanical systems from identified state space realizations. Part I: theoretical discussions. J Eng Mech 129(5): 477-488. doi:10.1061/(ASCE)0733-9399(2003)129:5(477)
Moreno-García P, Araújo dos Santos JV, Lopes H (2014) A new technique to optimize the use of mode shape derivatives to localize damage in laminated composite plates. Compos Struct 108:548-554

Mottershead JE, Friswell MI (1993) Model updating in structural dynamics. J Sound Vib 167:347-357

Mottershead JE, Link M, Friswell MI (2011) The sensitivity method in finite element model updating: a tutorial. Mech Syst Signal Process 25:2275-2296

Neugebauer R, Scheffler C, Wabner M, Schulten M (2010) Advanced state space modeling of non-proportional damped machine tool mechanics. CIRP J Manufact Sci Technol 3:8-13

Rao SS (2011) The finite element method in engineering. Elsevier Butterworth-Heinemann, UK

Reddy M, Swarnamani S (2013) Structural damage identification using signal processing method. Int J Adv Struct Eng 5:1-5

Roy K, Ray-Chaudhuri S (2013) Fundamental mode shape and its derivatives in structural damage localization. J Sound Vib 332:5584-5593

Salawu OS (1997) Detection of structural damage through changes in frequency: a review. Eng Struct 19:718-723

Shi Z, Law S, Zhang L (2000) Damage localization by directly using incomplete mode shapes. J Eng Mech 126:656-660

Shiradhonkar SR, Shrikhande M (2011) Seismic damage detection in a building frame via finite element model updating. Comput Struct 89:2425-2438

Wu JR, Li QS (2006) Structural parameter identification and damage detection for a steel structure using a two-stage finite element model updating method. J Constr Steel Res 62:231-239

Yan YJ, Cheng L, Wu ZY, Yam LH (2007) Development in vibration-based structural damage detection technique. Mech Syst Signal Process 21:2198-2211

Yang YB, Chen YJ (2009) A new direct method for updating structural models based on measured modal data. Eng Struct $31: 32-42$ 